
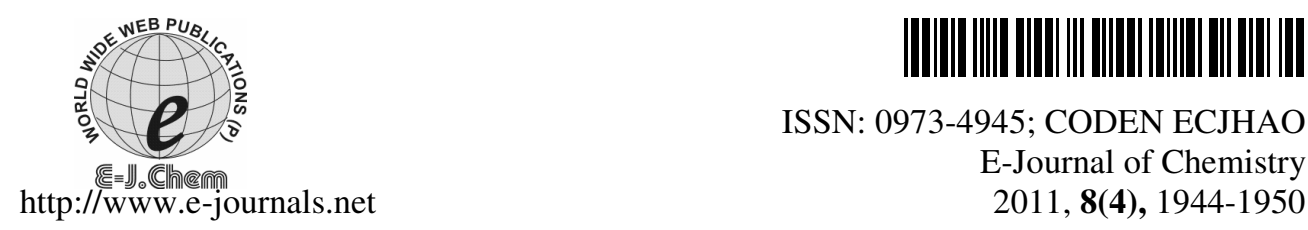

ISSN: 0973-4945; CODEN ECJHAO

E-Journal of Chemistry

2011, 8(4), 1944-1950

\title{
Chemical Studies on 3,6-Dichloropyridazine (Part 2)
}

\author{
N.M. ABD EL-SALAM*, Z.Y. AL SHOAIBI and G.A. AHMED ${ }^{\S}$ \\ *Department of Natural Sciences, Riyadh Community College \\ King Saud University, P.O.Box 28095, Riyadh-11437, Saudi Arabia \\ Organic Chemistry Department, Faculty of Pharmacy, Sana'a University, Egypt \\ ${ }^{\S}$ Chemistry Department, Faculty of Science, Zagazig University, Egypt \\ nelsalam@ksu.edu.sa
}

Received 30 November 2010; Accepted 22 January 2011

\begin{abstract}
Abstarct: 3,6-Dichloropyridazine (1) reacted with 2-aminophenol, phenylalanine, acetophenone hydrazone derivatives, acid hydrazide derivatives and amino-aromatic acids (anthranilic acid and 5-bromoanthranilic acid) and yield the compounds (2), (3), (4a,b), $(\mathbf{5 a}, \mathbf{b})$ and $(\mathbf{6 a}, \mathbf{b})$ respectively. Reaction of compounds $(\mathbf{5 a}, \mathbf{b})$ with acid hydrazide gave $(\mathbf{8} \mathbf{a}, \mathbf{b})$. Also, compounds $(\mathbf{6} \mathbf{a}, \mathbf{b})$ reacted with aromatic amino acid and gave (7a-c).
\end{abstract}

Keywords: Ditriazolopyridazine, Triazolopyridazine, Benzoimidazolopyridazine, Imidazolopyridazine

\section{Introduction}

The present work is an extension to our studies on the preparation of some fused heterocyclic compounds using 3,6-dichloropyridazine as a starting material ${ }^{1}$. Many derivatives of pyridazine have been synthesized when compound (1) is allowed to react with oxygen $^{2,3}$, sulfur ${ }^{4}$, nitroge ${ }^{5,6}$ and halogen nucleophile ${ }^{7-9}$. It has been pointed out that they possess extremely excellent biological activity ${ }^{10}$.

2-Chloro- and 2,6-dichloropyridazine are mainly found as medical and agricultural drug intermediate and pyridazine derivatives have been extensive therapeutic potential ${ }^{11}$ and other medical and technological applications ${ }^{12}$ as antibodies and antihypertensive agents ${ }^{13,14}$.

\section{Experimental}

All melting points are uncorrected and were determined on Gallenkamp electric melting point apparatus. IR spectra ( $\mathrm{KBr}$ disc) were recorded on a FT/IR-400 spectrophotometer (Perkin Elmer). ${ }^{1} \mathrm{H}$ NMR spectra were recorded on a varian-300 (DMS- $\mathrm{d}_{6}$ ) solution. Chemicals shifts are reported as $\delta$ values relative to tetramethylsilane (TMS) as internal reference. The elemental analyses were carried out at micro analytical center, Cairo University. 
Reaction of compound (1) with 2-aminophenol; formation of compound (2)

A mixture of compound (1) (0.01 mole) and 2-aminophenol (0.01 mole) or (0.02 mole) in DMF $(20 \mathrm{~mL})$ was heated under reflux for $7 \mathrm{~h}$. The solid obtained upon dilution with water, filtered off and crystallized from ethanol to give compound (2) as brown crystals (Table 2).

Reaction of compound (1) with L-phenylalanine; formation of compound (3)

A mixture of compound (1) ( 0.01 mole) and $L$-phenylalanine ( 0.01 mole) or $(0.02$ mole) was heated in oil bath at $180-200{ }^{\circ} \mathrm{C}$ for $3 \mathrm{~h}$. The reaction product was washed with water and crystallized from ethanol into (3) (Table 2).

Reaction of compound (1) with acetophenonehydrazone derivatives; formation of compound $(\mathbf{4 a}, \boldsymbol{b})$

A mixture of compound (1) $(0.01 \mathrm{~mole})$ and acetophenonehydrazone derivative $(0.01 \mathrm{~mol})$, was heated in oil bath at $160-180{ }^{\circ} \mathrm{C}$ for $4 \mathrm{~h}$. The solid formed after cooling was collected and recrystallzien from the proper solvent into compounds $(\mathbf{4 a}, \mathbf{b})$ (Table 2).

Reaction of compound (1) with acid hydrazides; formation of compound $(\mathbf{5} \boldsymbol{a}, \boldsymbol{b})$

A mixture of compound (1) (0.01 mole) and acid hydrazides, namely (benzoylhydrazine and 4-aminobenzoylhydrazine) ( $0.01 \mathrm{~mole})$ in $20 \mathrm{~mL} n$-butanol was heated under reflux for $8 \mathrm{~h}$; the reaction mixture was left to cool, the solid produced was collected and recrystallized from the proper solvent into compounds (5a,b) (Table 2).

Reaction of compounds $(\mathbf{5} \boldsymbol{a}, \boldsymbol{b})$ with acid hydrazides; formation of compounds $(\mathbf{8} \boldsymbol{a}-\boldsymbol{b})$

The above experiment was repeated replacing compound (1) by compounds (5a,b) (Table 2).

Reaction of compound (1) with aromatic amino acids; formation of compounds $(\boldsymbol{6} \boldsymbol{a}, \boldsymbol{b})$

Heat under reflux in $20 \mathrm{~mL} n$-butanol a mixture of compound (1) $(0.01 \mathrm{~mole})$ and aromatic amino acids namely (anthranilic acid and 5-bromoanthranilc acid) $(0.01 \mathrm{~mole})$ for $7 \mathrm{~h}$. The solid produced after cooling was collected, dried and recrystallized from the proper solvent into compounds (6a,b) (Table 2).

Reaction of compounds $(\mathbf{6} \boldsymbol{a}, \boldsymbol{b})$ with aromatic amino acids; formation of compounds $(\mathbf{7} \boldsymbol{a}, \boldsymbol{b})$

The above experiment was repeated replacing compound (1) by compounds (6a,b) (Table 2).

\section{Results and Discussion}

When compound (1) was allowed to react with orthoaminophenol in refluxing butanol it afforded the chlorobenzoimidazolopyridazine (2) (Scheme 1). The structure of compound (2) was confirmed from its correct analytical and spectral analysis (Table 1 and 2). Also, compound (1) reacted with phenylalanine by heating in oil bath at $160-180{ }^{\circ} \mathrm{C}$ it yield imidazolo pyridazine derivative (3) (Scheme 2). The chemical structure of compound (3) was elucidated from its correct analytical and spectral data (Table $1 \& 2$ ).

Heating a mixture of compound (1) and acetophenone hydrazone derivatives in an oil bath at 180-200 ${ }^{\circ} \mathbf{C}$ afforded the triazolopyridazine derivatives (4a,b) (Scheme 1). The structure of compounds (4a,b) was proved from their correct elemental and spectroscopic analysis (Table 1\&2).

On the other hand, when compound (1) (0.01 mole) was allowed to react with aroylhydrazine $(0.01$ mole) it yields the triazolopyridazine derivatives $(\mathbf{5 a}, \mathbf{b})$, which reacted with another molecule of aroylhydrazine afforded the compounds (8a-c) (Scheme 1). The structure of compounds $(\mathbf{5 a}, \mathbf{b})$ and $(\mathbf{8 a}-\mathbf{c})$ was confirmed from their correct elemental and spectral analysis (Table 1 and 2). 


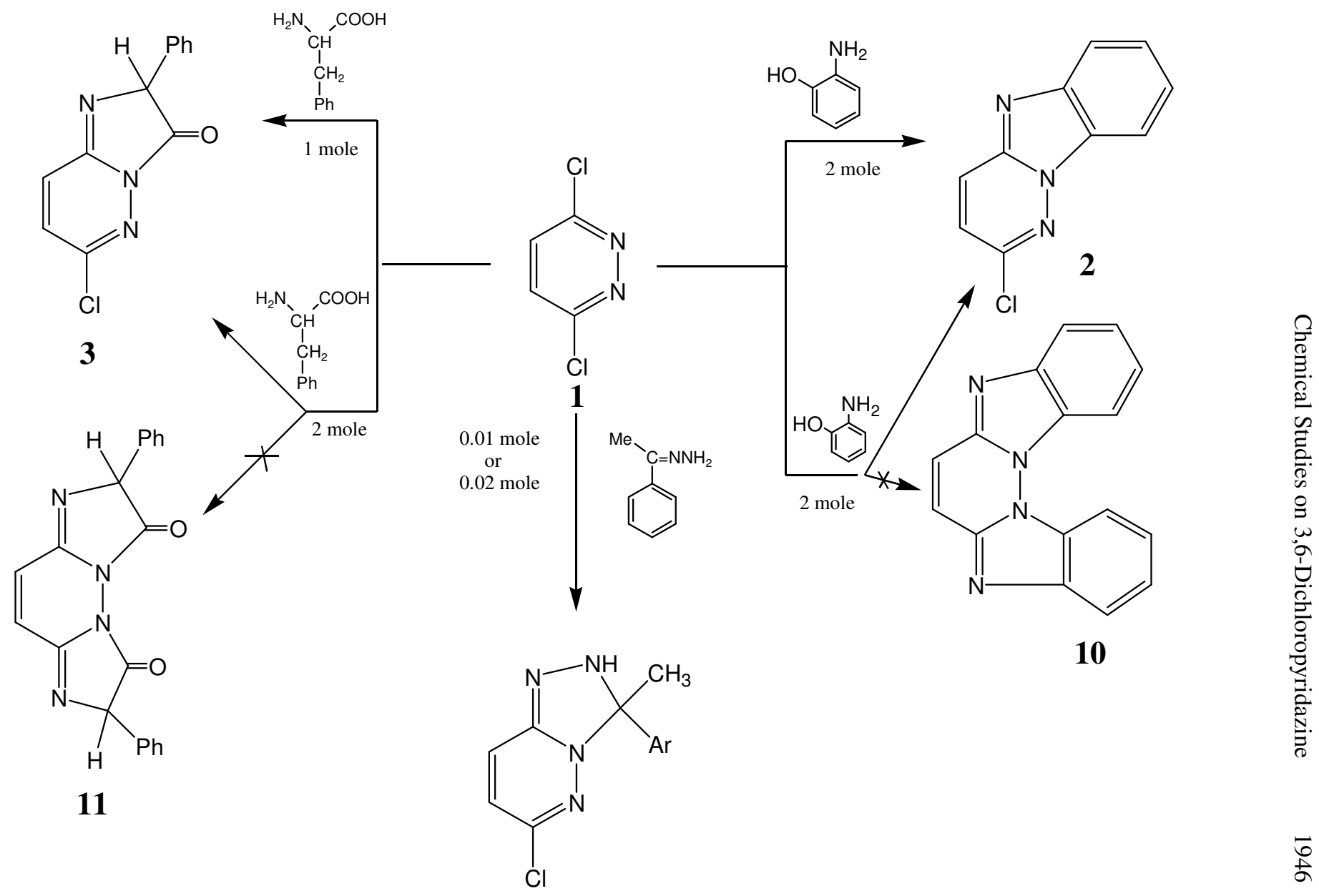

4a,b

a, $\mathrm{Ar}=\mathrm{C}_{6} \mathrm{H}_{5}{ }^{-}$

b, $\mathrm{Ar}=3-\mathrm{NO}_{2}-\mathrm{C}_{6} \mathrm{H}_{4}$ 


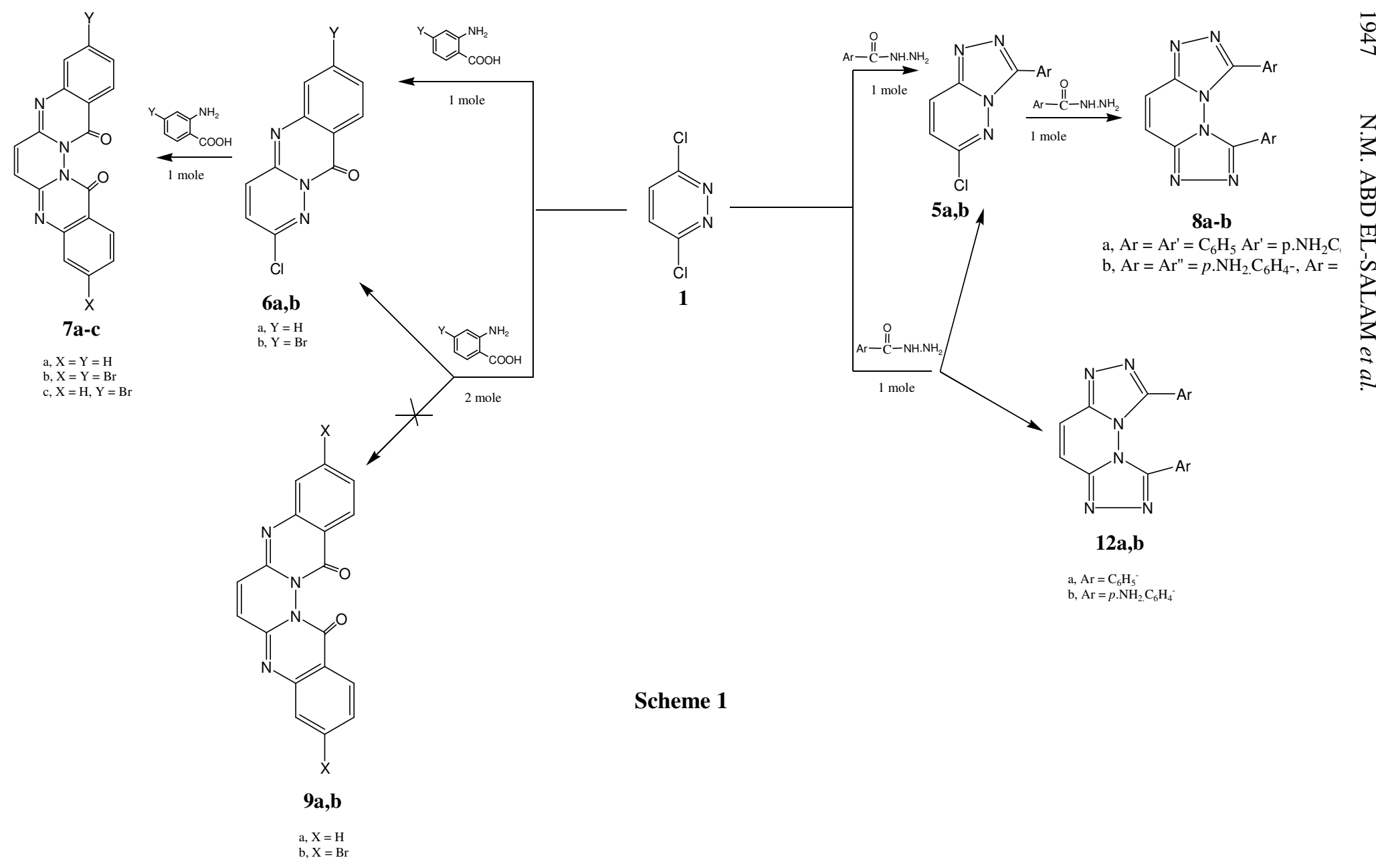


Reaction of compound (1) (0.01 mole) with aroylhydrazine (0.02 mole) afforded the triazolopyridazine derivatives $(\mathbf{5 a}, \mathbf{b})$ and not the expected ditrazolopyridazine derivative (12a-c) (Scheme 1).

Furthermore, when $(0.01$ mole $)$ of compound (1) was heated with $(0.01$ mole $)$ or ( 0.02 mole) of acetophenonehydrazone derivatives in oil bath at $180-200{ }^{\circ} \mathrm{C}$ for $3 \mathrm{~h}$, it from the triazolopyridazine derivatives $(\mathbf{4 a}, \mathbf{b})$ (Scheme 1). The structure of compounds $(\mathbf{4 a}, \mathbf{b})$ was elucidated by elemental and spectroscopic analysis (Table 1 and 2).

Also, compound (1), (0.01 mole reacted with aromatic amino acids $(0.01$ mole $)$ and gave benzimidazolopyridazine derivatives $(\mathbf{6 a}, \mathbf{b})$ (Scheme 1), which reacted with aromatic amino acids giving the polynuclear compounds (7a-c) (Scheme 1). The structure of $(\mathbf{5 a}, \mathbf{b})$ and $(\mathbf{7 a - c})$ was confirmed from their correct analytical and spectral data (Tables 1 and 2), but when compound (1) (0.01 mole) was allowed to react with aromatic amino acids $(0.02$ mole) it produce the compounds $(\mathbf{6 a}, \mathbf{b})$ and not the expected compounds (9a-c).

Table 1. Physical properties of the prepared compounds

\begin{tabular}{|c|c|c|c|c|c|c|c|}
\hline \multirow{2}{*}{$\begin{array}{l}\text { Compd. } \\
\text { No. }\end{array}$} & \multirow{2}{*}{ m.p. ${ }^{\circ} \mathrm{C}$} & \multirow{2}{*}{ Yield, \% } & \multirow{2}{*}{$\begin{array}{l}\text { Solvent of } \\
\text { cryst. }\end{array}$} & \multirow{2}{*}{$\begin{array}{l}\text { Mol. formula } \\
\text { (M.wt) }\end{array}$} & \multicolumn{3}{|c|}{$\begin{array}{l}\text { Elemental analysis } \\
\text { calcd./found }\end{array}$} \\
\hline & & & & & $\mathrm{C}$ & $\mathrm{H}$ & $\mathrm{N}$ \\
\hline \multirow{2}{*}{1} & \multirow{2}{*}{$69-70$} & \multirow{2}{*}{60} & \multirow{2}{*}{$\begin{array}{l}\text { Petroleum } \\
\text { ether }\end{array}$} & \multirow{2}{*}{$\begin{array}{c}\mathrm{C}_{4} \mathrm{H}_{2} \mathrm{Cl}_{2} \mathrm{~N}_{2} \\
148.98\end{array}$} & 32.25 & 1.35 & 18.80 \\
\hline & & & & & 32.21 & 1.33 & 18.79 \\
\hline \multirow{2}{*}{2} & \multirow{2}{*}{$130-132$} & \multirow{2}{*}{70} & \multirow{2}{*}{ Et-OH } & \multirow{2}{*}{$\begin{array}{c}\mathrm{C}_{10} \mathrm{H}_{6} \mathrm{ClN}_{3} \\
203.63\end{array}$} & 58.93 & 2.94 & 20.62 \\
\hline & & & & & 58.90 & 2.92 & 20.60 \\
\hline \multirow{2}{*}{3} & \multirow{2}{*}{$198-200$} & \multirow{2}{*}{75} & \multirow{2}{*}{ Et-OH } & \multirow{2}{*}{$\begin{array}{c}\mathrm{C}_{14} \mathrm{H}_{12} \mathrm{ClN}_{3} \mathrm{O} \\
273.72\end{array}$} & 61.37 & 4.38 & 15.34 \\
\hline & & & & & 61.35 & 4.35 & 15.30 \\
\hline \multirow{2}{*}{$\mathbf{4 a}$} & \multirow{2}{*}{$200-202$} & \multirow{2}{*}{70} & \multirow{2}{*}{ Et-OH } & \multirow{2}{*}{$\begin{array}{c}\mathrm{C}_{13} \mathrm{H}_{13} \mathrm{ClN}_{4} \mathrm{O} \\
276.08\end{array}$} & 56.50 & 4.70 & 20.28 \\
\hline & & & & & 56.48 & 4.68 & 20.26 \\
\hline \multirow{2}{*}{$4 b$} & \multirow{2}{*}{$200-205$} & \multirow{2}{*}{80} & \multirow{2}{*}{ Et-OH } & \multirow{2}{*}{$\begin{array}{c}\mathrm{C}_{12} \mathrm{H}_{10} \mathrm{ClN}_{5} \mathrm{O}_{2} \\
291.5\end{array}$} & 49.39 & 3.43 & 24.01 \\
\hline & & & & & 49.35 & 3.40 & 23.96 \\
\hline \multirow{2}{*}{$5 \mathbf{a}$} & \multirow{2}{*}{$230-231$} & 60 & $\mathrm{Et}-\mathrm{OH}$ & $\mathrm{C}_{11} \mathrm{H}_{7} \mathrm{ClN}_{4}$ & 57.22 & 3.04 & 24.27 \\
\hline & & & $2-11$ & 230.65 & 57.20 & 3.01 & 24.25 \\
\hline $5 b$ & $235-237$ & 80 & $\mathrm{Et}-\mathrm{OH}$ & $\mathrm{C}_{11} \mathrm{H}_{8} \mathrm{ClN}_{5}$ & 53.73 & 3.25 & 28.49 \\
\hline & & & & 245.67 & 53.71 & 3.23 & 28.46 \\
\hline $6 a$ & $238-240$ & 60 & Et-OH & $\mathrm{C}_{11} \mathrm{H}_{6} \mathrm{ClN}_{3} \mathrm{O}$ & 56.98 & 2.59 & 18.13 \\
\hline & & & & 231.64 & 56.95 & 2.57 & 18.10 \\
\hline $6 \mathrm{~b}$ & $260-262$ & 65 & $\mathrm{Et}-\mathrm{OH}$ & $\mathrm{C}_{11} \mathrm{H}_{5} \mathrm{BrClN}_{3} \mathrm{O}$ & 42.50 & 1.61 & 13.52 \\
\hline & & & & 310.53 & 42.48 & 1.60 & 13.50 \\
\hline 7 & $265-267$ & 65 & Et-OH & $\mathrm{C}_{18} \mathrm{H}_{9} \mathrm{BrN}_{4} \mathrm{O}_{2}$ & 54.93 & 2.28 & 14.24 \\
\hline & & & Lit-U11 & 393.19 & 54.92 & 2.28 & 14.22 \\
\hline 8 & $180-181$ & 60 & Et-OH & $\mathrm{C}_{18} \mathrm{H}_{13} \mathrm{~N}_{7}$ & 66.04 & 4.00 & 29.95 \\
\hline & & & & 327.34 & 66.3 & 3.98 & 29.92 \\
\hline
\end{tabular}


Table 2. Physical and spectral data of compounds (2-8)

\begin{tabular}{|c|c|c|}
\hline Compound & IR $\mathrm{cm}^{-1}$ & ${ }^{1} \mathrm{H}$ NMR, ppm \\
\hline \multirow[t]{3}{*}{2} & $1560(v \mathrm{C}=\mathrm{N})$ & $7.8(\mathrm{~d}, 1 \mathrm{H}, \mathrm{CH})$ \\
\hline & $1610(v \mathrm{C}=\mathrm{N})$ & $7.6(\mathrm{~d}, 1 \mathrm{H}, \mathrm{CH})$ \\
\hline & & $6.5-7.3(\mathrm{~m}, 4 \mathrm{H}$ arom $)$ \\
\hline \multirow[t]{4}{*}{3} & $1690($ v $\mathrm{C}=\mathrm{O})$ & $7.9(\mathrm{~d}, 1 \mathrm{H}, \mathrm{CH})$ \\
\hline & $1610(v \mathrm{C}=\mathrm{N})$ & $8.1(\mathrm{~d}, 1 \mathrm{H}, \mathrm{CH})$ \\
\hline & $1580(v \mathrm{C}=\mathrm{N})$ & $6.6-7.5(\mathrm{~m}, 5 \mathrm{H}$ arom $)$ \\
\hline & & $7.2(\mathrm{~s}, 1 \mathrm{H}$ imidazolone $)$ \\
\hline \multirow[t]{3}{*}{$4 \mathbf{a}$} & $1570(v \mathrm{C}=\mathrm{N})$ & $7.7(\mathrm{~d}, 1 \mathrm{H}, \mathrm{H})$ \\
\hline & $1530(v \mathrm{C}=\mathrm{N})$ & $7.8(\mathrm{~d}, 1 \mathrm{H}, \mathrm{CH})$ \\
\hline & $3320(\mathrm{v} \mathrm{NH})$ & $\begin{array}{l}6.5-7.3(\mathrm{~m}, 5 \mathrm{H} \text { arom and } 1 \mathrm{H} \text {, } \\
\text { Triazolyl })\end{array}$ \\
\hline \multirow[t]{4}{*}{$4 \mathbf{b}$} & $1550(v \mathrm{C}=\mathrm{N})$ & $7.9(\mathrm{~d}, 1 \mathrm{H}, \mathrm{CH})$ \\
\hline & $1580($ v $\mathrm{C}=\mathrm{N})$ & $8.1(\mathrm{~d}, 1 \mathrm{H}, \mathrm{CH})$ \\
\hline & $1460\left(v \mathrm{C}=\mathrm{NO}_{2}\right)$ & $6.5-7.4(\mathrm{~m}, 4 \mathrm{H}$ arom and $1 \mathrm{H}$, \\
\hline & $3320(v \mathrm{NH})$ & Triazolyl) \\
\hline \multirow{3}{*}{ 5a } & $1560($ v $\mathrm{C}=\mathrm{N})$ & $7.7(\mathrm{~d}, 1 \mathrm{H}, \mathrm{CH})$ \\
\hline & $1580(v \mathrm{C}=\mathrm{N})$ & $7.9(\mathrm{~d}, 1 \mathrm{H}, \mathrm{CH})$ \\
\hline & & $6.5-7.4(\mathrm{~m}, 5 \mathrm{H}$ arom \\
\hline \multirow[t]{2}{*}{$5 \mathbf{b}$} & $1675(v \mathrm{C}=\mathrm{N})$ & $8.0(\mathrm{~d}, 1 \mathrm{H}, \mathrm{CH}), 8.2(\mathrm{~d}, 1 \mathrm{H}$, \\
\hline & $1585(v \mathrm{C}=\mathrm{N})$ & $\begin{array}{l}\mathrm{CH}) 5.8\left(\text { broad, } 2 \mathrm{H}, \mathrm{NH}_{2}\right), \\
6.6-7.6(\mathrm{~m}, 4 \mathrm{H} \text { arom })\end{array}$ \\
\hline \multirow[t]{3}{*}{$6 \mathbf{a}$} & $1710(v \mathrm{C}=\mathrm{O})$ & $8.3(\mathrm{~d}, 1 \mathrm{H}, \mathrm{CH})$ \\
\hline & $1615(v \mathrm{C}=\mathrm{N})$ & $8.1(\mathrm{~d}, 1 \mathrm{H}, \mathrm{CH})$ \\
\hline & $1590(v \mathrm{C}=\mathrm{N})$ & $6.9-7.6(\mathrm{~m}, 4 \mathrm{H}$ arom $)$ \\
\hline \multirow[t]{3}{*}{$\mathbf{6 b}$} & $1725(v \mathrm{C}=\mathrm{O})$ & $8.2(\mathrm{~d}, 1 \mathrm{H}, \mathrm{CH})$ \\
\hline & $1610(v \mathrm{C}=\mathrm{N})$ & $8.0(\mathrm{~d}, 1 \mathrm{H}, \mathrm{CH})$ \\
\hline & $1580(v \mathrm{C}=\mathrm{N})$ & $6.8-7.4(\mathrm{~m}, 3 \mathrm{H}$ arom $)$ \\
\hline \multirow[t]{3}{*}{$7 \mathbf{a}$} & $1720(v \mathrm{C}=\mathrm{O})$ & $8.2(\mathrm{~d}, 1 \mathrm{H}, \mathrm{CH})$ \\
\hline & $1580(v \mathrm{C}=\mathrm{N})$ & $7.9(\mathrm{~d}, \mathrm{H}, \mathrm{CH}), 7.6(\mathrm{~s}, 1 \mathrm{H}$, \\
\hline & $1630(v \mathrm{C}=\mathrm{N})$ & pyridyl)6.8-7.4 (m, 7H arom) \\
\hline \multirow[t]{3}{*}{$7 b$} & $1700(v \mathrm{C}=\mathrm{O})$ & $8.2(\mathrm{~d}, 1 \mathrm{H}, \mathrm{CH})$ \\
\hline & $1610(v \mathrm{C}=\mathrm{N})$ & $8.1(\mathrm{~d}, 1 \mathrm{H}, \mathrm{CH}), 7.7(\mathrm{~s}, 1 \mathrm{H}$, \\
\hline & $1570(v \mathrm{C}=\mathrm{N})$ & pyridyl)6.5-7.3 (m, 7H arom) \\
\hline \multirow[t]{3}{*}{$7 \mathrm{c}$} & $1690(v \mathrm{C}=\mathrm{O})$ & $7.8(\mathrm{~d}, 1 \mathrm{H}, \mathrm{CH})$ \\
\hline & $1600(v \mathrm{C}=\mathrm{N})$ & $8.0(\mathrm{~d}, 1 \mathrm{H}, \mathrm{CH})$ \\
\hline & $1575(v \mathrm{C}=\mathrm{N})$ & $6.8-7.6(\mathrm{~m}, 7 \mathrm{H}$ arom $)$ \\
\hline \multirow[t]{3}{*}{$\mathbf{8 a}$} & $1610(v \mathrm{C}=\mathrm{N})$ & $8.3(\mathrm{~d}, 1 \mathrm{H}, \mathrm{CH}), 5.5-6$ (brand, \\
\hline & $1580(v \mathrm{C}=\mathrm{N})$ & $\left.2 \mathrm{H}, \mathrm{NH}_{2}\right) 8.0(\mathrm{~d}, \mathrm{H}, \mathrm{CH})$ \\
\hline & & $6.4-7.0(\mathrm{~m}, 9 \mathrm{H}$ arom $)$ \\
\hline \multirow[t]{4}{*}{$\mathbf{8 b}$} & $1590($ v $\mathrm{C}=\mathrm{N})$ & $7.9(\mathrm{~d}, 1 \mathrm{H}, \mathrm{CH})$ \\
\hline & $1620(v \mathrm{C}=\mathrm{N})$ & $8.1(\mathrm{~d}, 1 \mathrm{H}, \mathrm{CH})$ \\
\hline & $3310(v \mathrm{C}=\mathrm{N})$ & 5.4-6.0 (brand, $\left.2 \mathrm{H}, \mathrm{NH}_{2}\right)$ \\
\hline & & $6.7-7.3(\mathrm{~m}, 8 \mathrm{H}$ arom $)$ \\
\hline
\end{tabular}




\section{References}

1. Sherif M H, Ahmed G A, Elbahnasawy A A and Helal E O, J Am Sci., 2010, 6(11), 570.

2. Parrot I, Rival Y and Wermuth C G, Synthesis, 1999, 7, 1163-1168.

3. Huang J and Corey E J, Org Lett., 2003, 5(19), 3455-3458.

4. Parrot I, Wermuth C G and Hibert M, Tetrahedron Lett., 1999, 40, 7975-7978.

5. Rabisson P, Mekonnen B and Peet N P, Tetrahedron Lett., 2003, 44, 2919.

6. Ding S, Gray N S, Wu X, Ding Q and Schultz P G, J Am Chem Soc., 2002, 124, 1594.

7. Goodman A J, Stanforth S P and Tarbit B, Tetrahedron, 1999, 55, 15067.

8. Yamada T, Nobuhara Y, Shimamura H, Yoshihara K, Yamaguch A and Ohki M, Chem Pharm Bull., 1981, 29, 3433-3439.

9. Easmon J, Purstinger G, Heinisch G, Roth T, Fiebig H H, Holzer W, Jager W, Jenny M and Hofmann J, J Med Chem., 2001, 44(13), 2164-2171.

10. Hamdouchi C, Sauchez Martinez C, Gruber J, Del Prado M, Lopez Rubio A and Heiz B A, J Med Chem., 2003, 46, 4333-3341.

11. Tye S J, Wafford K A and Alack J R, J Med Chem., 2006, 49, 2660.

12. Luo S, MixWang B G and Cheng J P, Tetrahedron Lett., 2004, 45, 5171-5174.

13. Canmul and Hailes H C, Tetrahedron Lett., 2005, 46(47), 8125-8127.

14. Al-Awadi N A, Abdelhamed I A, Al Etaibi A M and Elnagdi M H, Synlett., 2007, 14, 2205-2208. 


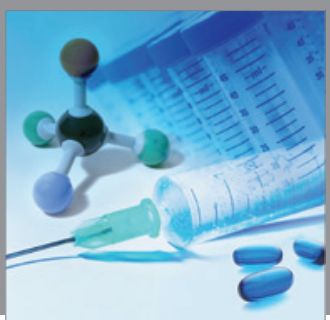

International Journal of

Medicinal Chemistry

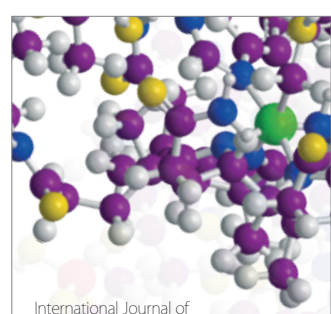

Carbohydrate Chemistry

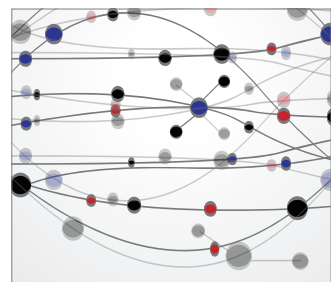

The Scientific World Journal
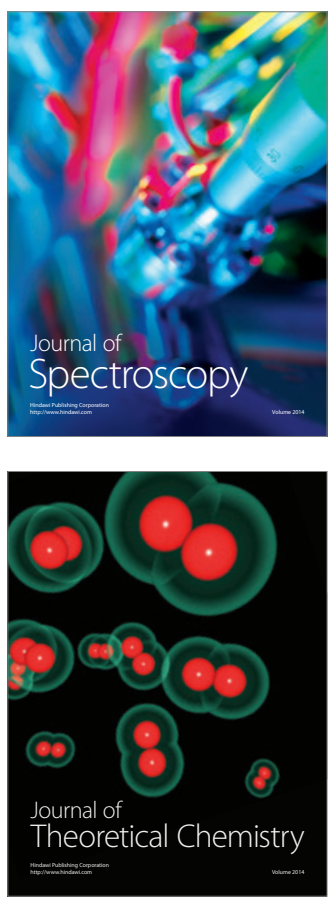
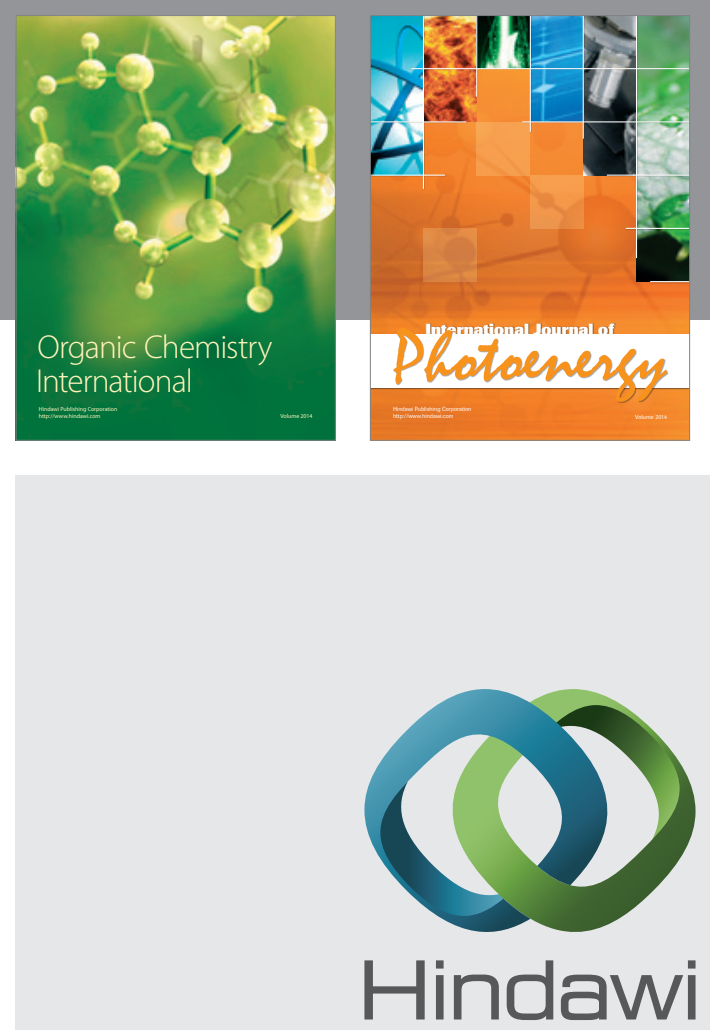

Submit your manuscripts at

http://www.hindawi.com
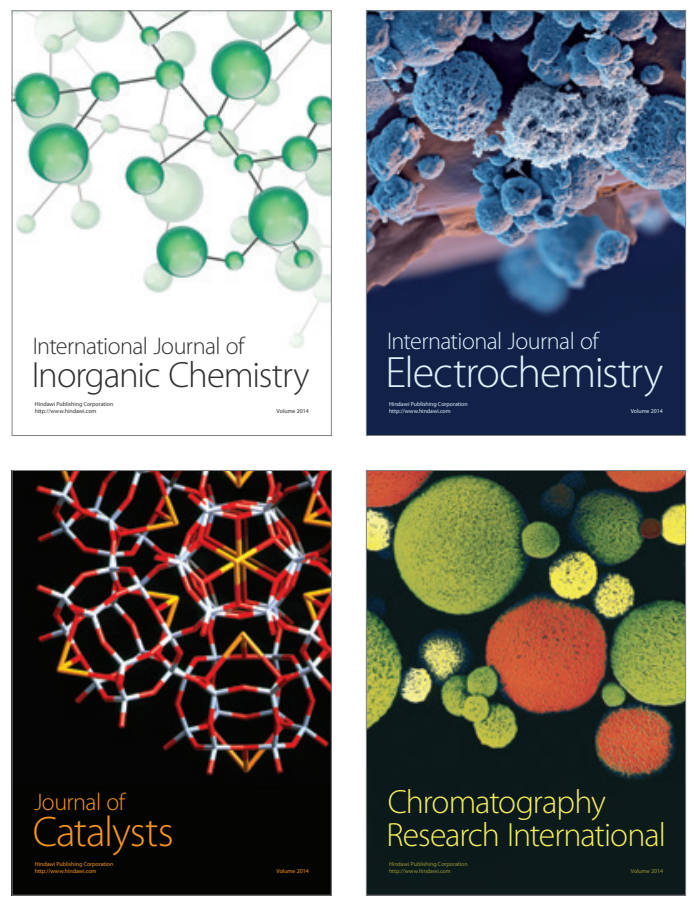
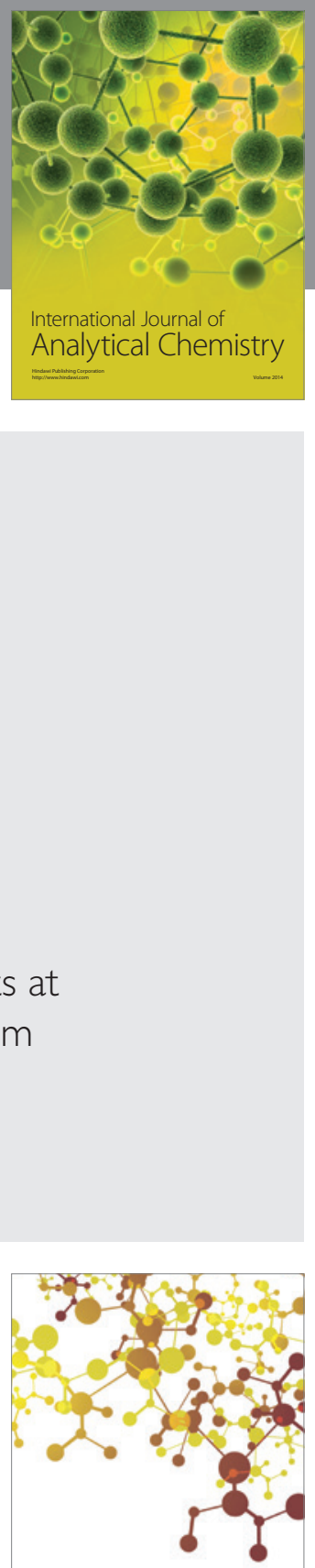

Journal of

Applied Chemistry
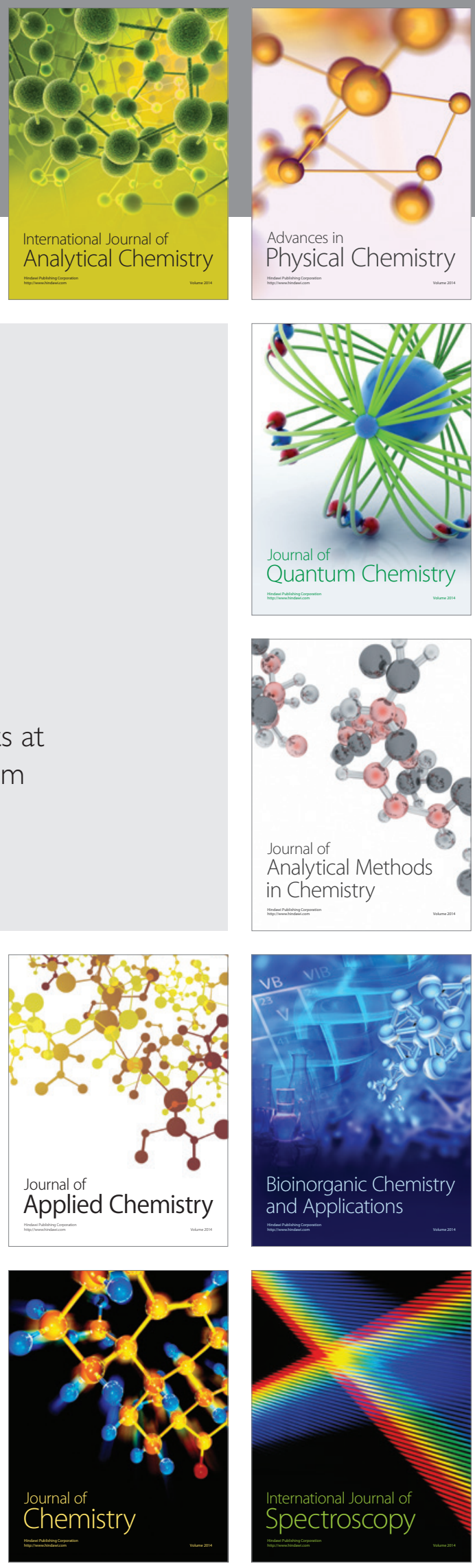